\title{
Embutidora de forrajes, alternativa de conservación de forrajes para la ganadería tropical, Santo Domingo de los Tsáchilas, Ecuador, 2009
}

\section{Autores:}

Ing. José Luis Cedeño Zambrano Ing. Carlos Samaniego 


\section{Resumen}

En el Ecuador existe la problemática de la baja producción de la ganadería por unidad de superficie (ha), ya que la mayoría de sistemas actuales se basan en el extensionismo lo que conlleva al mal uso del suelo, tala de bosques y subutilización del territorio agrícola. La falta de tecnificación, sistemas de riego hacen que los sistemas dependan directamente del factor ambiental que en varios años ha causado grandes pérdidas al sector ganadero del país. La embutidora de forrajes es una alternativa para la conservación de forrajes para alimentación de rumiantes necesaria para poder tener alimento durante todo el año y aprovechar eficientemente los subproductos de la industria y la producción invernal. Los resultados del proyecto demuestran que es una técnica eficiente de conservación ya que las pérdidas del producto ensilado son bajas (2\%). De los tratamientos evaluados el maíz, maíz más leguminosas, Brachiaria Decumbens, lodo de palma y mazorca de maíz cubren con los estándares para ser guardados lo que no sucede con el pasto Marafalfa que por su alto contenido de humedad y características propias no se puede ensilar en estado puro. La rentabilidad de este sistema de producción dependerá de la cantidad de forraje que produzca la hectárea sembrada y de los costos de los subproductos que se puedan utilizar para ensilar. Para que este sistema funcione de una manera eficiente es necesaria la mecanización y el aprovechamiento de terrenos planos además de la posibilidad de unificar capitales para que pequeños y medianos productores se asocien y formen unidades productivas más rentables y sostenibles en el tiempo. El panorama ecuatoriano, su clima, tipo de suelo y la tecnología adecuada más un manejo técnico adecuado tiene mejores perspectivas productivas que los sistemas de los países industrializados donde existe solo una cosecha al año mientras que en nuestro país se podrían realizar hasta tres al año lo que maximiza nuestro potencial.

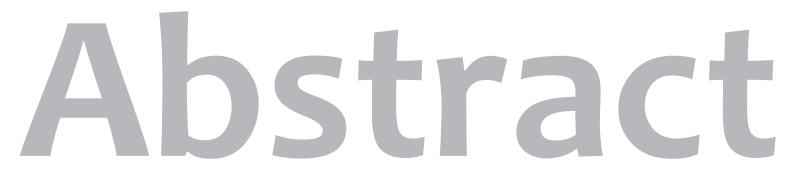

There is a low production of cattle per unit area in Ecuador because the most present systems are based on the expansion so it causes, the wrong or bad use of land, cutting down trees, and subutilization of the agricultural soil. The lacks of technology and water systems cause that they depend on environmental factors directly have caused huge losses to the cattle area of the country. The forage packer is an alternative for forage conservation to feed ruminates so they can have food all the year. In addition, it is necessary to profit the sub-products of the industry and the winter production efficiently.The results of the project show that is an efficient technique of conservation because the ensiled products are low (2\%).From the evaluated treatments the corn, maize more leguminous, bracharia decumbens, mud of palm and ear of corn carry out the standards to be kept which is not the same with the pasture mar alfalfa that has a high humid and own features it is not possible to ensile in pure conditions.The income of this production system will depend on quantity of forage that produces the grown hectare and the sub-products costs which can be used to ensile.So this system works in an efficient form, it is necessary the mechanization and utilization of flat terrains. In addition, there is the possibility to unify funds so that small and medium producers associate themselves and get more rentable and supportable productive units in the time. Ecuadorian outlook, its climate, the kind of soil, the right technology and a correct use of techniques give better productive perspectives than the technology of the industrialized countries where there is only a harvest in a year while in our country we could carry out up three in a year which maximizes our potential. 


\section{Introducción}

Según el Censo Nacional Agropecuario, SICA (2002), la región de Santo Domingo de los Colorados está conformada por una superficie de 309.000 hectáreas, a 400 metros, en promedio, sobre el nivel del mar. De las cuales el 66\% que representa 204.000 hectáreas están dedicadas a pastos cultivados con las siguientes variedades: Saboya, elefante, Bachearías, gramalote, miel, estrella, King Grass y Pueraria.

La distribución de las tierras se clasifica en la siguiente forma: productores pequeños $24 \%$, productores medianos el $68 \%$ y productores grandes el $8 \%$. La carga animal promedio de la región es de 1 cabeza por hectárea, la producción de leche promedio es de 1 litro por ha/día y de 300 gramos/ha/día de ganancia de peso. (SICA, 2002)

En el Ecuador la producción de pastos y forrajes en las zonas tropical y subtropical es sumamente rica en producción de alimentos, por lo cual existe gran desaprovechamiento de los mismos en la época de mayor producción (invierno) y escasez en varios meses por la sequía del verano por la falta de prevención y técnicas adecuadas para la conservación de forrajes. Esto ocasiona problemas económicos muy importantes de inestabilidad de precios y mortalidad de animales en las épocas críticas.

A pesar de que el uso de la conservación de forrajes y en especial de los ensilajes tiene una gran historia en los sistemas de producción animal en zonas templadas del mundo, que tienen la necesidad de guardar alimento para las épocas de escases, en el país no existe dicha cultura, solo en pocas haciendas de la sierra ecuatoriana se realiza. La técnica de ensilar el maíz es muy antigua, los primeros ensilajes de este cultivo se efectuaron en Estados Unidos en 1875, pero su uso masivo se implementó con el desarrollo de la maquinaria moderna que permitió cortar, picar y cargar el forraje mediante una sola operación.

En las zonas tropicales ha sido limitado o escaso su utilización, ha estado limitada a casos excepcionales en empresas ganaderas gra- des y particularmente con la industria lechera, no así a pequeños y medianos productores que son los que sufren los problemas de escases de forrajes en las épocas críticas. (FAO, 2001) El ensilado de cultivos forrajeros o de subproductos industriales podría aportar una importante contribución para optimizar el funcionamiento de los sistemas de producción animal en zonas tropicales y subtropicales, pero su empleo es todavía muy escaso Wilkins et al. (1999).

Según EL UNIVERSO, (2005), en la actualidad otros países tropicales y subtropicales manejan la tecnología adecuada para poder conservar los excedentes de la producción de pastos y forrajes aumentando la productividad y rentabilidad de sus ganaderías bovinas. En nuestro medio se ha hecho una escasa investigación al respecto teniendo grandes problemas técnicos y de costos en cuanto la conservación de forrajes y disponibilidad del mismo en épocas de escases.

La investigación buscó comparar y probar que la embutidora de forrajes es una técnica de conservación en el ensilaje de forrajes y subproductos para alimentación bovina, por otro lado, conocer cuál es el potencial de nuestros forrajes para estos sistemas de conservación y desarrollar técnicas eficientes para poder conservar los subproductos de la industria Ecuatoriana a gran escala en las épocas de sobreproducción. Todo lo anterior con la finalidad de ofrecer alternativas al sector ganadero para evitar los problemas en la época de escasez y así aumentar la productividad y mantenerla eficientemente.

Por lo anteriormente mencionado, se planteó los siguientes objetivos:

\section{Objetivos}

\section{○ Objetivo General}

Diseñar una alternativa de conservación forrajera y de suplementos alimenticios por medio de la embutidora de forrajes para la ganadería tropical de Santo Domingo de los Colorados. 


\section{Objetivos Específicos}

$\checkmark \quad$ Realizar un diagnóstico de la situación actual de la zona en el manejo y conservación de forrajes para la época seca

$\checkmark$ Comparar y fundamentar la embutidora de forrajes como alternativa de conservación por medio del ensilaje

$\checkmark \quad$ Implementar la embutidora de forrajes, determinar su eficiencia y medir el valor de sus productos como de la aceptación de la misma como alternativa de conservación forrajera

- Hipótesis

$\mathrm{Hi}=$ La embutidora de forraje servirá para conservar forrajes de una manera eficiente.

Ho = La embutidora de forraje no servirá para conservar forrajes de una manera eficiente.

\section{Materiales, Métodos y Procedimientos}

\section{- Ubicación}

La presente investigación se realizó en la Granja Experimental EL OASIS propiedad de la Universidad Tecnológico Equinoccial, ubicada en la Provincia Santo Domingo de los Tsáchila, Cantón Santo Domingo, Km 1 vía San Jacinto del Búa, margen izquierdo.

\section{- Situación Geográfica.}

La granja experimental Oasis, según la Estación Meteorológica de la Dirección de Aviación Civil (DAC-2007), se encuentra ubicada en las siguientes coordenadas:
Cuadro No 1.

Situación Geográfica de la Granja Experimental de la UTE

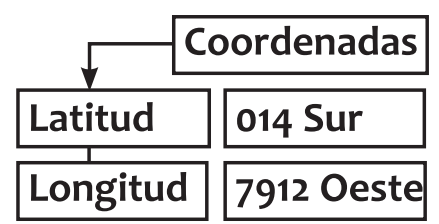

- Características Edafoclimáticas.

La zona de Santo Domingo presenta las siguientes características Edafoclimáticas, presentadas en el siguiente cuadro.

\section{Cuadro No 2}

\section{Características Edafoclimáticas de Santo Domingo}

\begin{tabular}{|c|c|c|c|}
\hline CARACTERÍSTICAS & MÁXIMO & MÍNIMO & \\
\hline Pluviosidad & & & $2718,2 \mathrm{~mm}$ anuales \\
\hline Temperatura & $26,08{ }^{\circ} \mathrm{C}$ & $20,14{ }^{\circ} \mathrm{C}$ & \\
\hline Humedad relativa & $99,26 \%$ & $78,91 \%$ & \\
\hline Luminosidad & & & 2-4 horas/día \\
\hline Clima & & & subtropical húmedo \\
\hline
\end{tabular}

Fuente: Dirección de Aviación Civil (DAC-2007).

- Factores de estudio

- $\quad$ Maíz Basilia

- $\quad$ Maís-INIAP-601

- Brachiaria Decumbens

- Marafalfa

- $\quad$ Lodo de Palma

- Mazorca de Maíz

- Maíz Brasilia sin mazorca asociada a frejol

- Maíz Brasilia sin mazorca asociada a Mucuna

- Maíz Brasilia sin mazorca asociada a Canabala 
- Variables

- Variables independientes

- Diferentes tipos de forrajes

- Aditivos para el ensilaje

- Variables dependientes

○ Tiempo de conservación

- Calidad nutricional

- Rendimiento

- Palatabilidad

\section{- Variables controladas}

- Materiales para la elaboración de la bolsa (tractor, maquina embutidora de forraje, bolsa plástica)

- Condiciones medioambientales iguales para todos los tratamientos.

- El mismo sitio del ensayo.

\section{Materiales e insumos}

\section{- Materiales}

$\begin{array}{llll}\circ \text { Bomba de mochila } & \circ & \text { Balanza } \\ \circ & \text { Jalones } & \circ & \text { Machetes } \\ \circ & \text { Machete } & \circ & \text { Cámara Fotográfica } \\ \circ & \text { Plástico } & \circ & \text { Filmadora } \\ \circ & \text { Tractor } & \circ & \text { Fundas de papel } \\ \circ & \text { Piola } & \circ & \text { Etiquetas } \\ \circ & \text { Cosechadora } & \circ & \text { Carretón } \\ \circ & \text { Ensiladora } & \circ & \text { Marcador } \\ \circ & \text { Palas } & \circ & \text { Flexómetro } \\ \circ & \text { Picadora } & \circ & \text { Cinta de Casset }\end{array}$

\section{Insumos}

\begin{tabular}{|c|c|c|c|}
\hline o & Semillas de maíz & o & Muriato de potasio \\
\hline O & Brachiaria & o & EM \\
\hline o & Lodo de Palma & ० & Melaza \\
\hline o & Mar alfalfa & ○ & Gramoxone \\
\hline o & Glifosato & ० & Amina 6 \\
\hline o & Pendimetalin & ○ & Kristalon \\
\hline o & Atrazina & ○ & Evergreen \\
\hline o & Cypermetrina & ○ & Methapac \\
\hline 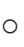 & Urea & ○ & Mancozeb \\
\hline & Diurco & & \\
\hline
\end{tabular}

Los tratamientos evaluados fueron los diferentes tipos de pastos con y sin aditivos descritos a continuación:

\section{Cuadro $\mathrm{No}_{3}$}

Descripción de los tratamientos evaluados

\begin{tabular}{|l|c|}
\hline \multicolumn{1}{|c|}{ Nombre } & Tratamiento \\
\hline Maíz Brasilia sin Aditivos & T1 \\
\hline Maíz Brasilia con Aditivos & T3 \\
\hline Maíz Iniap 601 sin Aditivos & T4 \\
\hline Maíz Iniap 601 con Aditivos & T5 \\
\hline Brachiaria Decumbens sin Aditivos & T6 \\
\hline Brachiaria Decumbens con Aditivos & T7 \\
\hline Lodo de Palma sin Aditivos & T8 \\
\hline Lodo de Palma con aditivos & T9 \\
\hline Mazorca de maíz & T10 \\
\hline Maíz Brasilia sin mazorca asociado con fréjol bolón & T11 \\
\hline Maíz Brasilia sin mazorca asociado con mucuna & T12 \\
\hline Maíz Brasilia sin mazorca asociado con canavalia & \\
\hline
\end{tabular}


Para la presente investigación se utilizará un diseño estadístico completamente al azar, (DCA) con tres observaciones. En caso de existir diferencias se utilizará la prueba de significancia de Tukey al 0,5\%.

\section{Cuadro No 4}

\section{Esquema del análisis estadístico de varianza empleado}

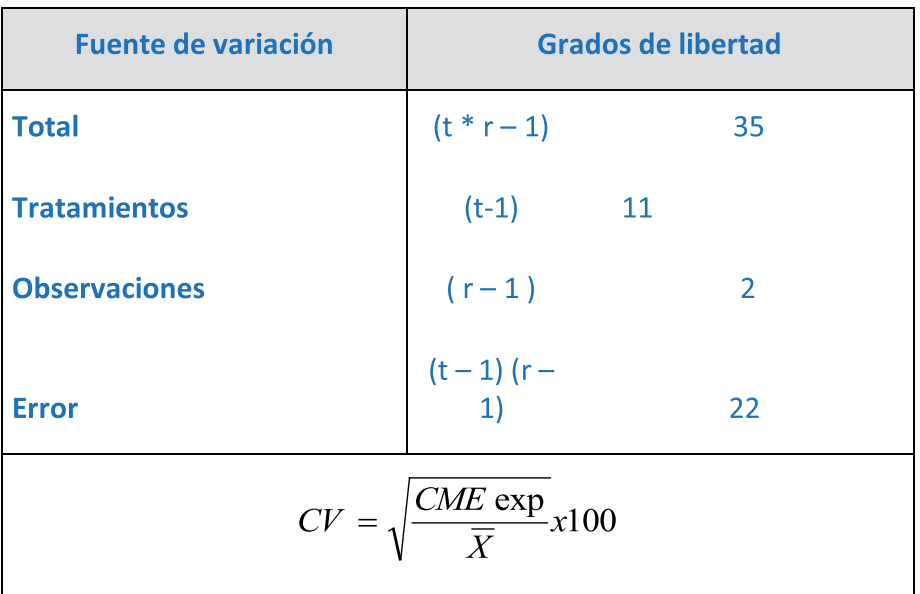

\section{- Manejo del experimento}

\section{- Manejo agronómico de la investigación}

\section{Maíz:}

Se realizó labores pre culturales como son el pase de rastra para la siembra del maíz (dos variedades), y su respectiva fertilización (Diurco 3 sacos).

Realizadas las labores pre culturales se procedió a la siembra a una densidad de 62500 plantas por ha. (0.20x0.80) de las dos variedades de maíz con una sembradora conectada al tractor.

Luego se procedió a realizar la fumigación de herbicida (Pendimetalin-1 litro $x$ tanque, atrazina-1 Kilo $x$ tanque, glifosato-1 litro $x$ tanque)

Se resembró a los 4 días de siembra un 30\% debido al problema de los pájaros. Se colocó cinta de casete para prevenir la presencia de aves, para la presencia de cogollero se controló con cipermetrina-300 $\mathrm{cm} 3 \times$ tanque.

Se realizó la primera fertilización con urea, tres sacos y muriato de potasio, dos sacos. La segunda fertilización se aplicó con urea, un saco y muriato de potasio- medio saco.

Se fumigó con Evergreen-1litro x tanque, Cipermetrina-300 cm $\mathrm{x}$ tanque, Methapac-1 sobre $\mathrm{x}$ tanque, Mancozeb-1 Kg x tanque, Kristalon-1 funda $x$ tanque, y se fumigó las calles con Gramoxone.

Se realizó la cosecha a los 103 días y se procedió a elaboración los primeros ensilajes con y sin aditivos.

\section{- Aditivos utilizados}

Se utilizó una mezcla de melaza, urea y microorganismos eficientes (EM) disueltos en agua a razón de dos litros de melaza, $1 \mathrm{~kg}$ de urea y 1 litro de EM por bomba de 20 litros. Se aplicó en los tratamientos realizados antes de ingresar el forraje a la bolsa de la embutidora.

\section{- Brachiaria Decumbens}

Se identificaron los potreros en los cuales se iba a trabajar y se realizó el corte de igualación con moto guadaña. Se fumigó con 2,4 D a razón de 2 litros por tanque para el control de malezas de hoja ancha. Se realizó luego una fertilización química con UREA y muriato de potasio a razón de tres y uno respectivamente.

Se cosechó la Brachiaria Decumbens a los 60 días, luego de haber realizado el corte de igualación, y se procedió a realizar los ensilajes con y sin aditivos.

\section{- Marafalfa:}

Se identificó los potreros con los cuales se iba a trabajar y se realizó el corte de igualación. Realizándose dos fertilizaciones con Urea y Muriato de potasio a dosis de cuatro y uno sacos (50kg/saco) 
por hectárea respectivamente.

Se cosechó la Marafalfa a los 56 días y se llevó a laboratorio obteniendo lo cosechado con el $82.14 \%$ de humedad. Tomándose la decisión de secarse durante siete días y se elaboró el ensilaje el cual no produjo un resultado por el alto contenido de humedad existente ya que el mismo tuvo un proceso de putrefacción.

De acuerdo con estos resultados, se tomó la decisión de excluirlo del análisis estadístico.

\section{- Lodo de Palma:}

El lodo de palma se obtuvo en la planta extractora de aceite de palma, se tomó una muestra para sacar humedad en laboratorio obteniendo $80.93 \%$ de humedad. Luego se elaboró el ensilaje con y sin aditivos.

\section{- Asocio con fréjol bolón, mucuna y canavalia}

Se planificó la siembra de frejol bolón, mucuna y canavalia en un cultivo de maíz variedad Brasilia destinado para baby corn con el fin de evaluar su potencial en silo con la embutidora de forraje. Se sembraron los materiales quince días después de la siembra del maíz, realizándose las mismas actividades culturales antes enunciadas en el cultivo de maíz con diferencia única en la densidad de plantas ya que esta fue de 80000 plantas/ha. Se cosechó el baby corn a partir del día 64 de la siembra durante quince días consecutivos. Igualmente, se cosechó el follaje del maíz y sus asociaciones el día 105 después de la siembra.

\section{- Proceso de ensilado}

Para el proceso de ensilado se utilizaron dos tipos de cosechadoras picadoras, accionadas por el toma de fuerza del tractor, para poder identificar la mejor calidad y velocidad de las mismas. Con un tractor adicional se realizó la actividad de transporte de los carretones con el forraje hasta el sitio definitivo de la funda silo. El lugar donde se colocó la funda fue plano, limpio de palos y objetos corto punzantes.
Con un tercer tractor se colocó la funda en la embutidora y se realizó el embutido de las mismas, con la ayuda de personal para la descarga del carretón. En el caso de los tratamientos con aditivos se utilizó una bomba de mochila para asperjar el forraje en la entrada a la embutidora.

\section{Figura No1.}

\section{Cosechadora picadora para maíz y forrajes (tipo A)}

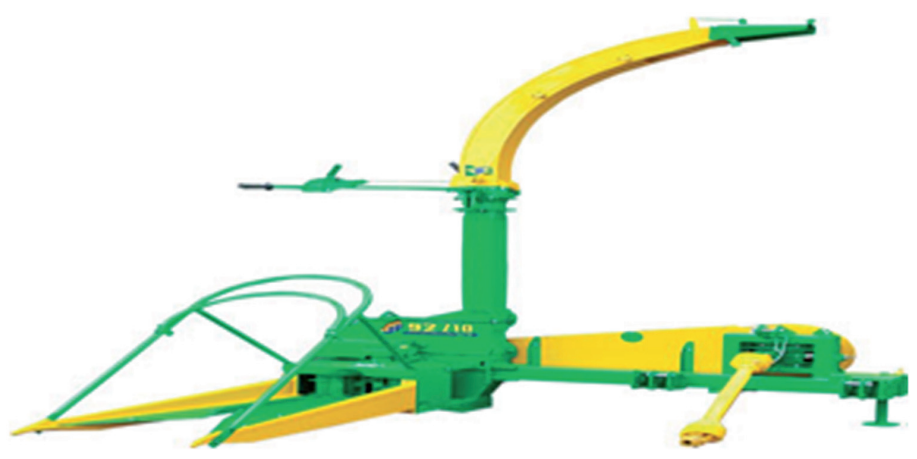

Figura No 2

\section{Cosechadora picadora forrajes (tipo B)}

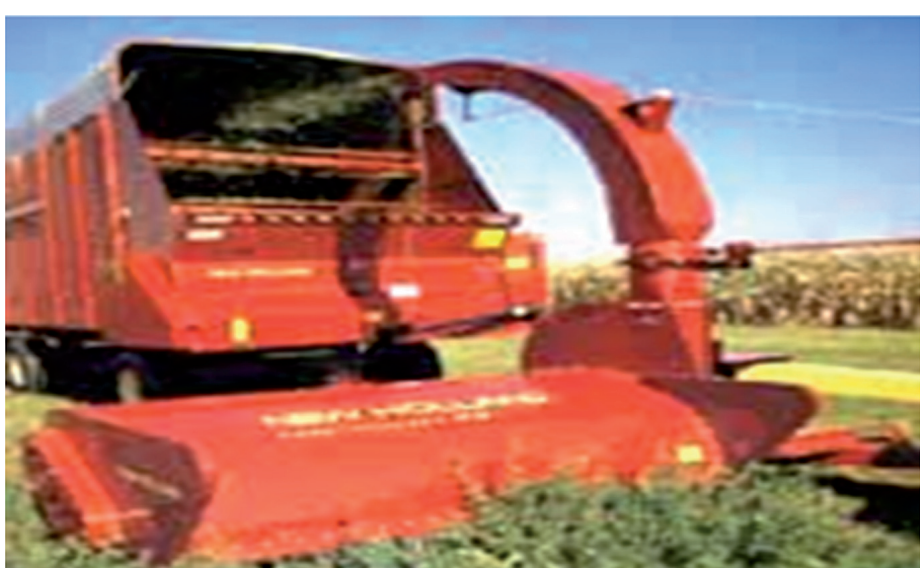

- Manejo de Laboratorio

Las muestras recolectadas de los diferentes tipos de ensilados fueron llevadas al laboratorio de la Universidad Tecnológica Equinoccial para determinar la composición bromatológica de cada uno de ellos en cuanto a humedad, proteína, fibra, grasa, ceniza, extracto libre de nitrógeno y energía bruta. 


\section{Resultados y Discusión}

\section{- Humedad}

En el análisis de varianza existen diferencias altamente significativas entre los tratamientos en lo que respecta a humedad existiendo cinco rangos de significancia. El tratamiento de menor humedad fue el de mazorca de maíz con el 59,83\% y el de mayor humedad fue el de maíz Brasilia para producción de baby corn sin mazorca con asociación de mucuna 86,24\%. El valor del coeficiente de variación fue de 5.41 $\%$, lo cual garantiza la confiabilidad de los datos reportados.

Según Boschini (2000), la materia seca del forraje óptima para ensilar es oscila entre el 25 al $35 \%$ (Plantas maduras). Se puede ensilar forrajes frescos con un mínimo de 20 \% de materia seca (Plantas jóvenes) y con un máximo de $40 \%$ (Plantas viejas). Cuando el contenido de materia seca es bajo, la masa ensilada se compacta excesivamente. El exceso de agua presente en el forraje hace que el proceso de ensilaje sea frío. Se deben agregar materiales secos en capas.

Cuando el contenido de materia seca es alto, la masa ensilada no se compacta fácilmente. Esto permite que la masa ensilada contenga mucho aire (oxígeno) y el proceso de ensilaje es sobrecalentado. Se debe humedecer con agua antes de compactar.

La clasificación de los ensilajes por la humedad según Boschini (2000) es:

\footnotetext{
- 69 a $71 \%$ (excelente calidad)

- entre $67-75 \%$ (bueno)

- más de $79 \%$ (mala calidad)

- menos $61 \%$ (mala calidad)
}

De acuerdo a los análisis de laboratorio (Cuadro No 5), los tra- tamientos $\mathrm{T} 1, \mathrm{~T} 2, \mathrm{~T} 3, \mathrm{~T} 4, \mathrm{~T} 7$, y $\mathrm{T} 8$ presentaron excelente calidad, los tratamientos T5 y T6, buena calidad y los tratamientos T9, T10, T11 y T12, mala calidad. A pesar de que la clasificación de Boschini indica la clasificación de mala calidad los datos de $\mathrm{pH}$, olor y color demuestran características aceptables y palatables para los animales. Todo esto indica que el silo fue realizado de buena forma sin presencia de oxígeno.

\section{Cuadro No 5}

Humedad, pH, producción por ciclo y características organolépti-

cas

\begin{tabular}{|l|c|c|c|c|c|c|c|c|}
\hline \multicolumn{1}{|c|}{ Nombre } & Trata. & Humedad & \multicolumn{2}{|c|}{$\mathrm{pH}$} & Olor & Color & palatabilidad \\
\hline Maíz Brasilia sin Aditivos & $\mathrm{T} 1$ & 71,38 & $\mathrm{ABC}$ & 3,8 & $\mathrm{ABCD}$ & agradable & $\begin{array}{l}\text { amarillo } \\
\text { verdoso }\end{array}$ & positiva \\
\hline Maíz Brasilia con Aditivos & $\mathrm{T} 2$ & 71,26 & $\mathrm{ABC}$ & 3,83 & $\mathrm{ABCD}$ & agradable & $\begin{array}{l}\text { Amarillo } \\
\text { verdoso }\end{array}$ & positiva \\
\hline Maíz Iniap 601 sin Aditivos & $\mathrm{T} 3$ & 70,56 & $\mathrm{ABC}$ & 3,79 & $\mathrm{ABCD}$ & agradable & $\begin{array}{l}\text { Amarillo } \\
\text { verdoso }\end{array}$ & positiva \\
\hline Maíz Iniap 601 con Aditivos & $\mathrm{T} 4$ & 60,96 & $\mathrm{AB}$ & 4,06 & $\mathrm{D}$ & agradable & $\begin{array}{l}\text { Amarillo } \\
\text { verdoso }\end{array}$ & positiva \\
\hline $\begin{array}{l}\text { Brachiaria Decumbens sin } \\
\text { Aditivos }\end{array}$ & $\mathrm{T} 5$ & 74,99 & $\mathrm{CD}$ & 4,66 & $\mathrm{E}$ & agradable & $\begin{array}{l}\text { amarillo } \\
\text { verdoso }\end{array}$ & positiva \\
\hline $\begin{array}{l}\text { Brachiaria Decumbens con } \\
\text { Aditivos }\end{array}$ & $\mathrm{T} 6$ & 72,79 & $\mathrm{C}$ & 4,63 & $\mathrm{E}$ & agradable & $\begin{array}{l}\text { amarillo } \\
\text { verdoso }\end{array}$ & positiva \\
\hline Lodo de Palma sin Aditivos & $\mathrm{T} 7$ & 71,57 & $\mathrm{BC}$ & 4,02 & $\mathrm{CD}$ & agradable & $\begin{array}{l}\text { amarillo } \\
\text { verdoso }\end{array}$ & positiva \\
\hline Lodo de Palma con aditivos & $\mathrm{T} 8$ & 70,43 & $\mathrm{ABC}$ & 4,02 & $\mathrm{CD}$ & agradable & $\begin{array}{l}\text { amarillo } \\
\text { verdoso }\end{array}$ & positiva \\
\hline Mazorca de maíz & $\mathrm{T} 9$ & 59,83 & $\mathrm{~A}$ & 3,87 & $\mathrm{BCD}$ & agradable & $\begin{array}{l}\text { amarillo } \\
\text { verdoso }\end{array}$ & positiva \\
\hline $\begin{array}{l}\text { Maíz Bras. sin m. asoc. con } \\
\text { fréjol bolón }\end{array}$ & $\mathrm{T} 10$ & 85,62 & $\mathrm{D}$ & 3,32 & $\mathrm{~A}$ & agradable & $\begin{array}{l}\text { amarillo } \\
\text { verdoso }\end{array}$ & positiva \\
\hline $\begin{array}{l}\text { Maíz Bras. sin m. asoc. con } \\
\text { mucuna }\end{array}$ & $\mathrm{T} 11$ & 86,24 & $\mathrm{D}$ & 3,52 & $\mathrm{ABC}$ & agradable & $\begin{array}{l}\text { amarillo } \\
\text { verdoso }\end{array}$ & positiva \\
\hline $\begin{array}{l}\text { Maíz Bras. sin m. asoc. con } \\
\text { canavalia }\end{array}$ & $\mathrm{T} 12$ & 81,45 & $\mathrm{CD}$ & 3,47 & $\mathrm{AB}$ & agradable & $\begin{array}{l}\text { amarillo } \\
\text { verdoso }\end{array}$ & positiva \\
\hline Coeficiente de variación (CV) & & 5,41 & 4,49 & & & \\
\hline
\end{tabular}

Diferentes letras demuestran diferencias significativas entre tratamientos

\section{$\mathrm{pH}$}

El análisis de varianza para el pH se observa en el Cuadro No 5, en el cual se observó diferencias altamente significativas entre tratamientos. El tratamiento con el menor pH es el T9 y el de mayor el T5. El valor del coeficiente de variación para esta variable fue de 4,49\%, con lo cual se garantiza los resultados de la investigación.

Stefanie J.W.H. citado por Bragachini (2008) señala que en periodo de fermentación el $\mathrm{pH}$ del ensilado por efecto de las bacterias que producen ácido láctico y otros ácidos bajará a valores entre 3,8 a 5,0. De manera general, el pH determinado en los tratamientos 
estudiados varía entre 3,32 y 4,66, lo que sugiere que los rangos establecidos en el presente trabajo están dentro de los parámetros recomendados para obtener ensilajes de buena calidad y así lograr una buena fermentación.

La aptitud al ensilaje del maíz, Brachiaria y lodo de palma africana es buena debido a que no le faltan carbohidratos para ser transformados en ácido láctico, presenta un bajo poder tampón que permite que el pH baje rápidamente y porque al ensilar el contenido en materia seca es elevado. Esto indica que el ensilaje se realizó en un medio anaeróbico lo que demuestra que el proceso de ensilaje en la bolsa por medio de la embutidora de forrajes funciona de manera eficiente.

\section{- Características organolépticas, Olor, Color, Palatabilidad}

Las características organolépticas de color, olor y la palatabilidad en los animales se encontraron en los rangos recomendados ya que existió en todos los tratamientos un olor agradable a fermentación, un color verde amarillo. En el caso de la palatabilidad los animales consumieron los ensilajes.

En una de las repeticiones del tratamiento de INIAP con aditivos existió un problema de pudrición este debido al rompimiento del plástico de algún tipo de animal posiblemente rata lo que ocasionó que una buena proporción del silo tenga entrada de aire y por lo cual exista putrefacción.

\section{Cuadro No 6}

Diferencias entre tratamientos en fibra, proteína, grasa, ceniza, extracto libre de nitrógeno (ELNN) y energía bruta (EB) utilizando la prueba de Tukey al 0,05\%

\begin{tabular}{|c|c|c|c|c|c|c|c|c|c|c|c|c|c|}
\hline NOMBRE & $\mathrm{T}$. & Fibra & (\%) & Proteín & $a(\%)$ & Grasa & (\%) & Ceniza & (\%) & ELNN & (\%) & EB (Kcal) & /kg) \\
\hline Maíz Brasilia sin Aditivos & T1 & 21,38 & $A B C D$ & 8,75 & A & 5,45 & $A B C$ & 21,68 & C & 42,73 & $A B$ & 3805,09 & A \\
\hline Maíz Brasilia con Aditivos & $\mathrm{T} 2$ & 25,15 & $B C D$ & 8,31 & A & 3,43 & A & 23,84 & C & 39,27 & $A B$ & 3649,29 & A \\
\hline $\begin{array}{l}\text { Maíz Iniap } 601 \text { sin } \\
\text { Aditivos }\end{array}$ & T3 & 16,30 & $A B$ & 8,86 & A & 6,55 & $A B C$ & 23,80 & C & 44,49 & $A B$ & 3724,54 & \\
\hline $\begin{array}{l}\text { Maíz Iniap } 601 \text { con } \\
\text { Aditivos }\end{array}$ & T4 & 20,11 & $A B C$ & 8,66 & A & 4,64 & $A B$ & 9,18 & $A B$ & 57,42 & D & 4260,36 & B \\
\hline $\begin{array}{l}\text { Brachiaria Decumbens } \\
\sin \text { Aditivos }\end{array}$ & T5 & 27,36 & $C D$ & 8,24 & A & 5,76 & $A B C$ & 12,50 & B & 46,13 & $B C$ & 4238,47 & B \\
\hline $\begin{array}{l}\text { Brachiaria Decumbens } \\
\text { con Aditivos }\end{array}$ & T6 & 31,23 & D & 7,35 & A & 5,57 & $A B C$ & 11,50 & B & 44,36 & $A B$ & 4288,81 & $B C$ \\
\hline $\begin{array}{l}\text { Lodo de Palma sin } \\
\text { Aditivos }\end{array}$ & $\mathrm{T} 7$ & 19,92 & $A B C$ & 16,73 & C & 15,79 & D & 13,82 & B & 33,74 & A & 4747,88 & EF \\
\hline $\begin{array}{l}\text { Lodo de Palma con } \\
\text { aditivos }\end{array}$ & T8 & 18,91 & $A B C$ & 15,47 & $B C$ & 15,72 & D & 13,86 & B & 36,04 & $A B$ & 4708,98 & EF \\
\hline Mazorca de maíz & T9 & 11,50 & A & 10,70 & $A B$ & 16,30 & D & 5,40 & A & 56,10 & $C D$ & 4918,21 & $\mathrm{~F}$ \\
\hline $\begin{array}{l}\text { Maíz Bras. sin m. asoc. } \\
\text { con fréjol bolón }\end{array}$ & T10 & 23,72 & $B C D$ & 11,84 & $A B C$ & 8,55 & C & 8,72 & $A B$ & 47,17 & $B C D$ & 4557,94 & DE \\
\hline $\begin{array}{l}\text { Maíz Bras. sin m. asoc. } \\
\text { con mucuna }\end{array}$ & $\mathrm{T} 11$ & 22,97 & $B C D$ & 14,70 & $B C$ & 7,53 & $B C$ & 11,55 & B & 43,25 & $A B$ & 4443,07 & $B C D$ \\
\hline $\begin{array}{l}\text { Maíz Bras. sin m. asoc. } \\
\text { con canavalia }\end{array}$ & T12 & 23,87 & $B C D$ & 11,38 & $A B$ & 8,40 & $B C$ & 9,47 & $A B$ & 46,88 & $B C D$ & 4512,71 & $\mathrm{CDE}$ \\
\hline $\begin{array}{l}\text { Coeficiente de variación } \\
\text { (CV) }\end{array}$ & & 16,9 & & 16,4 & & 14,9 & & 13,85 & & 8,5 & & 1,97 & \\
\hline
\end{tabular}

Diferentes letras demuestran diferencias significativas entre tratamientos

\section{Valor nutritivo de los diferentes tratamientos}

\section{- Fibra}

En el Cuadro No 6, se aprecia para el contenido de fibra de los ensilados de maíz, en el cual se observó diferencias altamente significativas entre tratamientos. Existen cinco rangos de significancia obteniendo el menor porcentaje de fibra el de la mazorca de maíz T9 con $11,5 \%$ y la mayor el pasto Brachiaria Decumbens con 31,23\%. El valor del coeficiente de variación fue de 16,90 \%, lo cual garantiza la confiabilidad de los datos reportados.

Se puede diferenciar fácilmente que los porcentajes mayores de fibra se encuentran en el pasto Brachiaria Decumbens, un pasto tropical en el cual la cantidad de fibra dependerá de la edad de corte del mismo, en el caso de las dos variedades de maíz con la presencia y ausencia de aditivos el porcentaje de fibra es el esperado al igual que en el caso de los tratamientos con Taraya de maíz en asocio con las leguminosas, los cuales son parámetros esperados. 
En el caso de lodo de palma africana y mazorca de maíz se los puede considerar como alimentos no fibrosos por sus bajos contenidos de fibra y altos contenidos de grasa lo que hace que estos alimentos sean ricos en energía. Existiendo la necesidad de realizar mayor cantidad de evaluaciones en alimentación de animales.

\section{- Proteína}

El análisis de varianza para el contenido de proteína se observa en el Cuadro No 6, en el cual se observó diferencias altamente significativas entre tratamientos. Existen tres rangos de significancia obteniendo el menor porcentaje de proteína el de pasto Brachiaria T6 con 7,35\% mientras que el mayor es el de lodo de palma T7 con $16,73 \%$. El valor del coeficiente de variación fue de 16,45 \%, lo cual garantiza la confiabilidad de los datos reportados.

En el caso de los valores de los tratamientos T1, T2, T3, y T4 de los diferentes tratamientos con maíz los valores concuerdan con las investigaciones de Zambrano 2007, Riofrio 2007 y Toscano 2007 lo que demuestra que el ensilaje mantiene la calidad nutricional del alimento sin disminución de su calidad. En el caso del pasto Brachiaria el valor de $7,35 \%$ con aditivos y $8,24 \%$ sin aditivos es mayor a lo reportado por Riofrio de 6,12\% lo que posiblemente se deba al diferente manejo de los pastos y a la de edad de corte.

En una investigación que realizaron en la "Finca la María" ubicado en la ciudad de Riobamba, donde se evaluó la cantidad forrajera de maíz empleando los siguientes términos: T1 (5 kg de forraje de maíz); T2 (4.5kg de forraje de maíz +0.5 Kg de forraje de soya); T3 (4.okg de forraje de maíz $+1.0 \mathrm{~kg}$ de forraje de soya) y T4 (3.5kg de forraje de maíz +1.5kg de forraje de soya), con la adición del 3\% de melaza. Obteniendo que T3 $(M M+20 S)$, obtuvo el valor más alto para el contenido de proteína bruta (14,7\%). Esto puede explicarse por el alto contenido proteínico que tiene esta leguminosa y riqueza en hidratos de carbono que tiene el maíz. Lo cual coincide con los tratamientos T11, T12 y T13 que tienen 11,84 \%; 14,7 \%; 11,38 \% respectivamente que demuestra que la adición de leguminosas al forraje de maíz aumenta la cantidad de proteína en el ensilaje. Según Rodriguez (2001) el maíz húmedo o también llamado mazorca de maíz se puede guardar en el sistema de bolsa sin ningún inconveniente manteniendo la calidad nutritiva del mismo lo que concuerda con la presente investigación.

\section{- Grasa}

En el Cuadro No 6, se aprecia para el contenido de grasa de los diferentes tratamientos, se observó diferencias altamente significativas entre tratamientos. Existen cuatro rangos de significancia obteniendo el menor \% de grasa el Brachiaria T6 con 5,57 \% y la mayor la mazorca de maíz con 16,3\%.

El valor del coeficiente de variación fue de 14,95 \%, lo cual garantiza la confiabilidad de los datos reportados.

En el caso de grasa existen valores importantes que demuestran la calidad de los diferentes tratamientos en lo que respecta a las reservas energéticas que podrían proporcionar en la alimentación animal. Los valores de grasa de la mazorca de maíz y lodo de palma con y sin aditivos demuestran su altísimo potencial en la alimentación animal de rumiantes.

\section{- Ceniza}

Para el contenido de ceniza en el Cuadro No 6, se aprecia de los diferentes tratamientos, diferencias altamente significativas entre tratamientos. Existen tres rangos de significancia obteniendo el menor porcentaje de ceniza la mazorca de maíz con 5,40 \% y la mayor significancia el maíz Brasilia con aditivos, T2, del 23,84\%. El valor del coeficiente de variación fue de 13,85 \%, lo cual garantiza la confiabilidad de los datos reportados.

\section{- Extracto libre de nitrógeno, ELNN}

En el mismo cuadro antes mencionado, para el contenido de extracto libre de nitrógeno de los diferentes tratamientos se aprecia- 
ron diferencias altamente significativas entre tratamientos. Existen cuatro rangos de significancia obteniendo el menor porcentaje de ceniza el de lodo de palma africana sin aditivos T7 con 33,74\% y el mayor maíz INIAP 601 con aditivos T4 con 57,42\%.

El valor del coeficiente de variación fue de 8,5\%, lo cual garantiza la confiabilidad de los datos reportados.

\section{- Energía bruta, EB}

En el Cuadro No 6, para el contenido de Extracto libre de nitrógeno de los diferentes tratamientos, se apreciaron diferencias altamente significativas entre tratamientos. Existen seis rangos de significancia obteniendo el menor contenido de energía bruta el Maíz Brasilia con aditivos T2 con 3649,29 Kcal/kg y la mazorca de maíz con $4918 \mathrm{Kcal} / \mathrm{kg}$. El valor del coeficiente de variación fue de 1,97\%, lo cual garantiza la confiabilidad de los datos reportados.

El maíz es por excelencia un alimento energético tanto el grano como su forraje, en el caso de la mazorca de maíz T9 su mayor contenido de grano hace que la cantidad de energía sea mayor, mientras que los tratamientos T1, T2, T3, T4 que contenían el forraje de maíz más su grano tuvieron menores valores por tener menor cantidad de grano. Lo que lleva a la reflexión es que a pesar que los tratamientos T10, T11 y T12 no tengan grano poseen mayor cantidad de energía que los tratamientos con grano antes mencionados esto se debe a que estos poseen las asociaciones con leguminosas que ayuda a aumentar la cantidad de proteína y grasa lo cual se ve reflejado en la energía bruta.

La energía bruta es un parámetro que sirve para visualizar el potencial de los forrajes pero para conocer su real valor es necesario realizar pruebas en animales y conocer el aprovechamiento de los mismos por medio de energía digestible y energía metabolizable.

\section{- Producción de forraje}

La producción de los tratamientos T1, T2, T3 y T4 es similar, esta se realizó a los 105 días de siembra, necesitándose de 15 días para su respectiva adecuación en una posterior siembra, existiendo la posibilidad con la ayuda de riego en las épocas de sequia de realizar tres cultivos al año. Según Martinez \& Stanek en Argentina el promedio de producción por hectárea de ensilaje de maíz es de 35 toneladas por hectárea y un ciclo por año lo cual nos demuestra que por ciclo en Ecuador somos menos eficientes pero por producción por año se tiene la posibilidad de aumentar la producción y eficiencia del terreno.

Según Cattani (2005) el potencial de una hectárea de maíz ensilada en Argentina es de $40.000 \mathrm{~kg}$ de forraje verde que incluye $5.500 \mathrm{~kg}$ de grano de maíz, con 30\% de materia seca y 2,66 Mcal/EM/ kg MS que producirá $1.585 \mathrm{~kg}$ de carne lo que en Ecuador a precio de mercado \$0,65 USD por libra en pie daría \$2.266,25 USD, siendo esto un negocio lo suficientemente rentable como para invertir en la maquinaria necesaria.

A pesar de que no se han realizado investigaciones en alimentación animal utilizando como único ingrediente al ensilaje de maíz, existe un potencial en el Ecuador que puede ser explotado. En una investigación realizada por Riofrio en la finca EL OASIS de la UTE con un sistema semiestabulado incluyendo alimentación mixta en potrero con pasto Brachiaria y en estabulación con maíz, se obtuvieron ganancias de $0,82 \mathrm{~kg}$ día en animales de $250 \mathrm{~kg} / \mathrm{PV}$ lo que demuestra que existe el potencial para realizar engorde o producción de leche con este tipo de producto y obtener excelentes resultados de rentabilidad.

En el caso de los tratamientos T5 y T6 la producción de forraje es inferior a la producción esperada la cual es citada por la casa comercial Agripac que explica la producción de hasta 20 ton/MS/ha/año lo que posiblemente se deba al manejo de la pastura pero a su vez supera el promedio nacional de 0,7 UBA/ha.

En el caso de los tratamientos T 7 y T 8 correspondientes al lodo de palma africana, al ser un subproducto de adquisición en la época de 
abundancia (invierno) la alternativa de su conservación para épocas de escases ayuda al productor a prevenir y planificar su adquisición, mucho más al observar en los cuadros anteriores sus cualidades nutritivas las cuales tendrán que ser evaluadas en futuras investigaciones.

\section{Cuadro No 7}

Producción de forraje verde, materia seca por ciclo, año y carga animal posible

\begin{tabular}{|l|c|c|c|c|c|c|}
\hline \multicolumn{1}{|c|}{ Nombre } & Trat & $\begin{array}{c}\text { Producción/ } \\
\text { Ciclo (ton) }\end{array}$ & $\begin{array}{c}\text { Producción/ Año } \\
\text { (ton) }\end{array}$ & MS/ha/ciclo & MS/ha/año & UBA/año \\
\hline Maíz Brasilia sin Aditivos & $\mathrm{T} 1$ & 25,0 & 75,0 & 7,2 & 21,5 & 4,9 \\
\hline Maíz Brasilia con Aditivos & $\mathrm{T} 2$ & 25,0 & 75,0 & 7,2 & 21,6 & 4,9 \\
\hline Maíz Iniap 601 sin Aditivos & $\mathrm{T} 3$ & 26,0 & 78,0 & 7,7 & 23,0 & 5,2 \\
\hline Maíz Iniap 601 con Aditivos & $\mathrm{T} 4$ & 24,0 & 72,0 & 9,4 & 28,1 & 6,4 \\
\hline $\begin{array}{l}\text { Brachiaria Decumbens sin } \\
\text { Aditivos }\end{array}$ & $\mathrm{T} 5$ & 9,0 & 54,0 & 2,3 & 13,5 & 3,1 \\
\hline $\begin{array}{l}\text { Brachiaria Decumbens con } \\
\text { Aditivos }\end{array}$ & $\mathrm{T} 6$ & 9,0 & 54,0 & 2,4 & 14,7 & 3,4 \\
\hline Lodo de Palma sin Aditivos & $\mathrm{T} 7$ & - & - & - & - & - \\
\hline Lodo de Palma con aditivos & $\mathrm{T} 8$ & - & - & - & - & - \\
\hline Mazorca de maíz & $\mathrm{T} 9$ & 5,9 & 11,8 & 2,4 & 7,1 & - \\
\hline $\begin{array}{l}\text { Maíz Bras. sin m. asoc. con } \\
\text { fréjol bolón }\end{array}$ & $\mathrm{T} 10$ & 51,0 & 178,5 & 7,3 & 25,7 & 5,9 \\
\hline $\begin{array}{l}\text { Maíz Bras. sin m. asoc. con } \\
\text { mucuna }\end{array}$ & $\mathrm{T} 11$ & 47,0 & 164,5 & 6,5 & 22,6 & 5,2 \\
\hline $\begin{array}{l}\text { Maíz Bras. sin m. asoc. con } \\
\text { canavalia }\end{array}$ & $\mathrm{T} 12$ & 38,0 & 133,0 & 7,0 & 24,7 & 5,6 \\
\hline
\end{tabular}

En el caso del tratamiento T9, mazorca de maíz este fue realizado con las hojas de la mazorca y la tusa siendo este un impedimento para poder comparar con datos similares. Aunque a pesar de esto la investigación buscó la posibilidad de guardar esta para un futuro aprovechamiento incluso con un alto contenido de humedad se pudo realizar su conservación por medio del ensilaje en la funda de la embutidora.

En los tratamientos T10, T11 y T12 el potencial es mucho mayor ya que la producción por ha es mayor y los ciclos de producción son menores, esta se da a los 90 días su aprovechamiento. Existiendo la necesidad de igual manera de evaluar este potencial en los animales y así poder definir su utilización a gran escala. Con el potencial que expresa Cattani existe una gran expectativa en lo que se pueda realizar en ganancias de peso con estos tratamientos ya que su valor nutritivo aparentemente es mayor por el contenido de proteína que posee por contener leguminosas en su composición.
Como se puede observar la carga animal en Unidad Bovina Adulta (UBA) y su consumo calculado al $3 \%$ en su peso vivo es superior al promedio de la zona, si dotamos del 100\% de aprovechamiento de estos materiales.

Cabe destacar que los mismos se podrían utilizar como parte de un sistema nutricional y así aprovechar de una mejor manera su potencial como lo recomendado por Riofrio (2008).

\section{- Evaluación Económica}

Como se observa en el Cuadro 9. los costos por tonelada e ingresos dependen de la producción de forraje de los diferentes tratamientos mientras mayor sea su producción de toneladas por hectárea menor serán sus costos y viceversa. En el caso de los ingresos de todos los tratamientos se calcularon a razón del precio actual de mercado, en el caso de forraje a \$ 50 la tonelada, lo que corresponde al valor del henolaje en la serranía ecuatoriana y en el caso de mazorca de maíz a razón de \$176 la tonelada.

\section{Cuadro No 8}

\section{Costos e ingresos de los diferentes tratamientos}

\begin{tabular}{|c|c|c|c|c|c|c|c|c|c|}
\hline \multirow[b]{2}{*}{ Nombre } & \multirow[b]{2}{*}{ Trata. } & \multicolumn{4}{|c|}{ Costos } & \multicolumn{2}{|c|}{ Ingresos } & \multirow[b]{2}{*}{ Utilidad } & \multirow{2}{*}{\begin{tabular}{|c} 
Relación \\
B/C
\end{tabular}} \\
\hline & & ton & aditivo & Total & ha. & \begin{tabular}{|l|} 
Producto \\
\end{tabular} & Forraje & & \\
\hline Maíz Brasilia sin Aditivos & $\mathrm{T} 1$ & 43,6 & 0,0 & 43,6 & 1002,7 & \begin{tabular}{|c|}
- \\
\end{tabular} & 1650,0 & 647,3 & 1,6 \\
\hline Maíz Brasilia con Aditivos & $\mathrm{T} 2$ & 43,6 & 4,6 & 48,2 & 1118,2 & - & 1650,0 & 531,8 & 115 \\
\hline Maíz Iniap 601 sin Aditivos & T3 & 38,8 & 0,0 & 38,8 & 1009,3 & - & 1716,0 & 706,7 & 1 , \\
\hline Maíz Iniap 601 con Aditivos & T4 & 38,8 & 4,6 & 43,4 & 1129,4 & - & 1584,0 & 454,6 & \\
\hline $\begin{array}{l}\text { Brachiaria Decumbens sin } \\
\text { Aditivos }\end{array}$ & T5 & 39,3 & 0,0 & 39,3 & 364,0 & - & 594,0 & 230,0 & \\
\hline $\begin{array}{l}\text { Brachiaria Decumbens con } \\
\text { Aditivos }\end{array}$ & T6 & 39,3 & 4,6 & 44,0 & 405,6 & - & 594,0 & 188,4 & 1, \\
\hline Lodo de Palma sin Aditivos & $\mathrm{T7}$ & 29,5 & 0,0 & 29,5 & - & - & & & \\
\hline Lodo de Palma con aditivos & T8 & 29,5 & 4,6 & 34,1 & - & - & & & \\
\hline Mazorca de maíz & T9 & 109,7 & 0,0 & 109,7 & 603,2 & - & 1038,4 & 435,2 & \\
\hline $\begin{array}{l}\text { Maíz Bras. sin m. asoc. con } \\
\text { fréjol bolón }\end{array}$ & $\mathrm{T} 10$ & 22,5 & 0,0 & 22,5 & 1145,7 & 1230,0 & 3366,0 & 3450,3 & \\
\hline $\begin{array}{l}\text { Maíz Bras. } \sin \text { m. asoc. con } \\
\text { mucuna }\end{array}$ & & 24,0 & 0,0 & 24,0 & 1128,7 & 1181,0 & 3102,0 & 3154,3 & \\
\hline $\begin{array}{l}\text { Maíz Bras. sin m. asoc. con } \\
\text { canavalia }\end{array}$ & T12 & 28,8 & 0,0 & 28,8 & 1092,7 & 1248,0 & 2508,0 & 2663,3 & \\
\hline
\end{tabular}

Todos los tratamientos tienen una utilidad pero los tratamientos 10,11 y 12 tienen una utilidad representativa esto se debe a que tienen dos ingresos de recursos, el primero de la producción de baby corn y el segundo de la producción de forraje. En estos casos el ingreso de la venta de baby corn cubre los gastos totales de la confección 
de los silos quedando prácticamente extra y libre de costos la producción de forraje ensilado.

Sin duda quedan expectativas para el aprovechamiento de esta técnica del ensilaje ya que no existen en esta investigación datos de la utilidad en los animales y los beneficios que estos podrían dar en una explotación comercial de esta técnica. Cattani señala que en la producción de una ha de ensilaje de maíz se pueden obtener $1.585 \mathrm{~kg}$ de carne lo que en Ecuador a precio de \$0,65 la libra en pie (ASOGAN 2008) representaría un ingreso de $\$ 2266,55$ por producción de carne por hectárea, el cual es superior al de la venta del forraje.

\section{Conclusiones y Recomendaciones}

\section{Conclusiones}

1. El número de personas que realizan ensilajes en el Ecuador es insignificante debido a la falta de procesos eficientes y técnicos de esta forma de conservación.

2. La técnica de conservación de forrajes por medio del ensilaje con la ayuda de la embutidora de forrajes funciona de una manera eficiente y económicamente viable.

3. El maíz INIAP 601 y Brasilia y pasto Brachiaria decumbes responden muy bien al ensilaje en bolsas plásticas.

4. El asocio de leguminosas en la producción de baby corn ayuda a mejorar la calidad y cantidad del ensilaje y responde muy bien al ensilaje con la embutidora de forraje.

5. No se puede realizar ensilaje del pasto Marafalfa en estado puro por su alto contenido de humedad y características nutricionales.

6. Es viable realizar ensilaje de lodo de palma africana y mazorca de maíz con el sistema de la embutidora de forraje.
7. Las características nutricionales de los materiales ensilados se mantienen por un periodo de tiempo.

8. Es necesaria la unificación de capitales entre pequeños y medianos ganaderos para poder acceder a esta tecnología y aumentar la productividad por hectárea de terreno y poder competir a nivel internacional.

9. Existe un gran potencial en el Ecuador con el uso de la embutidora de forraje en diferentes sistemas de producción tanto para ganado de carne como de leche.

10. La embutidora de forrajes puede servir como un negocio de venta de forraje ensilado en la época de escases en el litoral ecuatoriano.

\section{Recomendaciones}

1. Se recomienda utilizar los ensilajes como medio de conservación de forrajes siempre y cuando los materiales a utilizar tengan características nutricionales adecuadas.

2. Se recomienda evaluar digestibilidad de los materiales y observar su disponibilidad para los animales.

3. Se necesita continuar con investigaciones de estos ensilajes en la producción animal en diferentes sistemas de producción.

4. Si se quisiera utilizar al pasto Marafalfa para la realización de silos se recomienda mezclarlo con forrajes con bajo contenido de humedad para poder equilibrar la misma.

5. Se recomienda la utilización de la cosechadora de forraje tipo A por tener un mejor picado y compactación en la bolsa permitiendo un mejor llenado de la misma y por ende una mejor fermentación.

6. Se necesita evitar al máximo las ratas y otros animales cerca a las fundas del silo ya que estos pueden realizar agujeros que 
dañarían el ensilaje.

7. Se recomienda a las entidades gubernamentales a apuntar a la tecnología y de esa forma poder mejorar la producción del productor ganadero.

\section{Bibliografía consultada}

1. Abdelhadi L. 2007. Utilización de los forrajes conservados de la boca del animal a la producción de leche y carne. 4 p.

2. Arcevedo. R., Guillermo. 2003 Nutrición de Ganado de Carne. Colombia: Angel Agro. 182p.

3. Becerra J.; Castaño M. 1998. Corrales L. Ensilaje sin maquinaria para zonas de laderas el trópico cálido. El Fondo Ganadero de Córdoba S. A. y CORPOICA. Colombia. 24 p.

4. Bernal. J. 1991 Pastos y Forrajes Tropicales. Producción y Manejo. Banco Ganadero, 2 Ed... 543p.

5. Bragachini M.; Cattani P. 2008. Eficiencia de cosecha de forraje conservado. Hacia la excelencia de conservación y utilización de forrajes de alta calidad. INTA, Proyecto PRECOP II. Gacetilla de Prensa. 6 p.

6. Bragachini M.; Cattani P. Gallardo M. Peiretti J. 2008. Forrajes conservados de alta calidad y aspectos relacionados al manejo nutricional. INTA. Argentina. 365 p.

7. Castignani H.; Zehnder R. 2004. La alimentación en el Tambo, pérdidas por suministro y aprovechamiento. INTA Rafaela. 3p.

8. Cattani P. 2007. Estrategias para la confección y utilización de silajes de maíz y sorgo. 7 p.

9. Cattani P. 2007. Forrajes conservados de alta calidad. 4 p.

10. Cattani P. 2008. Embolsado de forrajes. 50 p.
11. Cattani P. 2008. Las bases para el logro del éxito en el proceso de extracción y suministro de silaje. 3 p.

12. EL UNIVERSO. 2005. Problemas en la ganadería del Litoral Ecuatoriano. Octubre.

13. FAO. 1999. Uso del ensilaje en el trópico privilegiando opciones para pequeños campesinos. Memorias de la Conferencia de la FAO sobre Ensilajes en los Trópicos. 189 p

14. Fraga. R. 2005. Metodología de la investigación científica. Quito. Ecuador.

15. IDEAGRO, Grupo Industrial. 2007. SILO PRESS MA-80, Una buena calidad en el almacenamiento del alimento de su ganadería. Un excelente negocio. Disponible en www.ideagro.com.co. 8p.

16. Jim Linn, 2001. Necesidades Nutritivas del ganado vacuno lechero, resumen de las normas del NRC. Department of Animal Science. University of Minnesota. 24 p.

17. Malinarich, H. D. 2005. Moledora Embutidora M\&S, Alternativas de uso. Argentina. 22 p.

18. Martinez y Staneck. 2005. Embutidora de forraje, Manual de operación. Argentina. 19 p.

19. Martinez y Staneck. 2005. Soluciones embolsadas. Argentina $10 \mathrm{p}$.

20. Masushima, J. K. Alimentación de vacuno para carne. Zaragoza, España: Acribia, 1979. 142.p.

21. MINISTERIO DE AGRICULTURA Y DESARROLLO RURAL. 2006. Alternativas para enfrentar una sequia prolongada en la Ganadería prolongada. $38 \mathrm{p}$.

22. Rodríguez J. C. et. al. 2002. Almacenaje de Granos en bolsas plásticas: sistema Silobag. Informe final maíz. Fundación Ar- 
gentina. Delegación Buenos Aires Sur. 27 p.

23. Wattiaux M. 2000. Alimentos para vacas lecheras. Instituto Babcock para la Investigación y Desarrollo Internacional de la Industria Lechera, Universidad de Wisconsin-Madison. Revista Esenciales Lecheras. 4 p.

24. www.martinezystaneck.com. Embutidora de forrajes

25. www.sica.gov.

\section{El Investigador}

José Luis Cedeño Zambrano

jcedenio@ute.edu.ec

Ingeniero Agrónomo; Universidad

EARTH - Costa Rica, 2003

Maestría en Producción Animal

Tropical, Universidad Tecnológica

Equinoccial 